\title{
Biological Evaluation of Arylamine Mannich Base Derivatives with Potent In Vivo Activity as Potent Antichagasic Agents ${ }^{+}$
}

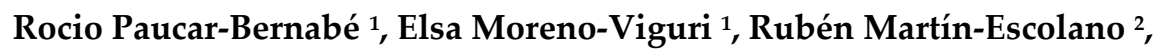 \\ Mery Santivañez-Veliz ${ }^{1}$, Amaya Azqueta ${ }^{3,4}$, Nuria Cirauqui ${ }^{5}$, Manuel Sánchez-Moreno ${ }^{2}$ and \\ Silvia Pérez-Silanes ${ }^{1, *}$
}

1 Department of Organic and Pharmaceutical Chemistry, Institute of Tropical Health, Universidad de Navarra, 31008 Pamplona, Spain; rpaucar@alumni.unav.es (R.P.-B.); emviguri@unav.es (E.M.-V.); msantivanez.1@alumni.unav.es (M.S.-V.)

2 Departamento de Parasitología, Instituto de Investigación Biosanitaria (ibs.GRANADA), Hospitales Universitarios de Granada/Universidad de Granada, 18014 Granada, Spain; martinescolano@ugr.es (R.M.-E.); msanchem@ugr.es (M.S.-M.)

3 Department of Pharmacology and Toxicology, Universidad de Navarra, Pamplona 31008, Spain; amazqueta@unav.es

4 IdiSNA, Navarra Institute for Health Research, Recinto de Complejo Hospitalario de Navarra, Pamplona 31008, Spain

5 Department of Pharmaceutical Sciences, Federal University of Rio de Janeiro, Rio de Janeiro 21949-900, Brazil; ncirauqu@gmail.com

* Correspondence: sperez@unav.es; Tel.: +34-948425600

+ Presented at the 1st Molecules Medicinal Chemistry Symposium, Barcelona, Spain, 8 September 2017.

Published: 18 October 2017

Chagas disease (CD) is a neglected tropical disease caused by the parasite Trypanosoma cruzi [1]. About 6-7 million people are infected worldwide, mainly in Latin America [1]. Benznidazole and Nifurtimox are the only available drugs for this disease but the problems with those drugs are related to their variable antiparasitic activity, the undesired side effects and long treatment duration among others [2]. Therefore, there is a great need for the development of new, effective, safe and affordable drugs for the treatment of $\mathrm{CD}$.

In this context, our group is focused on identifying new agents to fight against CD. So, twenty new derivatives were synthetized and tested in epimastigote, amastigote and trypomastigote forms in three different T. cruzi strains (SN3, Arequipa and Tulahuen). The cytotoxicity was also determined to establish their selectivity index (SI). The lead compound showed in vitro SI ranging from 99 to 258 times higher than Benznidazol in the amastigote form and from 333 to 2810 in the trypomastigote form of the parasites. The tested compounds in the SOS/umu screening test were non-genotoxic, whereas the reference drugs showed genotoxicity in the tested conditions. Regarding the studies of their mechanism of action, it seems that this family could be inhibitors of the Fe-SOD exclusive antioxidant defense trypanosomatids.

According to their in vitro biological activity and preliminary toxicological studies, four out of twenty derivatives were selected for an in vivo assay in a murine mice model. The in vivo acute model showed that the compounds decrease the parasitemia from the beginning of the treatment and parasites were not detected from day 25 post-infection. Moreover, none of the compounds showed reactivation after immunosuppression with the dose used with the reference drug (100 $\mathrm{mg} / \mathrm{kg}$ ) and the lead compounds showed no reactivation also at $50 \mathrm{mg} / \mathrm{kg}$ [3].

Considering the in vivo results, three out of four derivatives were selected for their mutagenicity evaluation and were non-mutagenic in the Ames test. Moreover, the absorption, distribution, metabolism, and excretion (ADME)/Tox and Pharmacokinetic (PK) evaluations are ongoing and the results will be presented in this congress. 
Up to now, these results have encouraged us to propose these compounds as promising molecules for developing new anti-Chagas agents and they will be transferred to an in vivo bioluminescence model in collaboration with the London School of Hygiene and Tropical Medicine.

Acknowledgments: M.J.-S. and R.P.-B. are indebted to the University of Navarra for a grant. A.A. thanks the Ministerio de Economía y Competitividad ("Ramón y Cajal" Programme, 2013) of the Spanish Government for personal support. R.M-E. is grateful for a FPU Grant FPU14/01537 from the Ministry of Education of Spain. This work has been carried out with the financial support of Fundación Caja Navarra (Project 70314), the Institute of Tropical Health from the University of Navarra (Project API-2011/01), and the former Spanish Ministry of Science and Innovation (MICINN) and now the Ministry of Economy and Competitiveness (MINECO) (Project Consolider Ingenio CSD2010-00065).

Author Contributions: R.P.-B., R.M.-E. and M.S.-V. carried out the experiments. E.M.-V., A.A. and N.C. wrote the manuscript and helped supervise the project. M.S.-M. and S.P.-S. supervised the project.

Conflicts of Interest: The authors declare no competing financial interest.

\section{References}

1. World Health Organization (WHO). Chagas Disease (American trypanosomiasis)/Fact Sheet $\mathrm{N}^{\circ} 340$. Available online: http://www.who.int/mediacentre/factsheets/fs340/en/ (accessed on 6 March 2017).

2. Paucar, R.; Moreno-Viguri, E.; Pérez-Silanes, S. Challenges in Chagas Disease Drug Discovery: A Review. Curr. Med. Chem. 2016, 23, 3154-3170.

3. Moreno-Viguri, E.; Jiménez-Montes, C.; Martín-Escolano, R.; Santivañez-Veliz, M.; Martin-Montes, A.; Azqueta, A.; Jimenez-Lopez, M.; Ledesma, S.Z.; Cirauqui, N.; de Ceráin, A.L.; et al. In Vitro and in Vivo Anti-Trypanosoma cruzi Activity of New Arylamine Mannich Base-Type Derivatives. J. Med. Chem. 2016, 59, 10929-10945.

(C) 2017 by the authors. Licensee MDPI, Basel, Switzerland. This article is an open access article distributed under the terms and conditions of the Creative Commons Attribution (CC BY) license (http://creativecommons.org/licenses/by/4.0/). 